\title{
BMJ Open Effect of the vancomycin minimum inhibitory concentration on clinical outcomes in patients with methicillin- susceptible Staphylococcus aureus bacteraemia: a systematic review and meta-analysis
}

Changcheng Shi, ${ }^{1}$ Jian Ye, ${ }^{2}$ Renjie Xu, ${ }^{3}$ Weizhong $\mathrm{Jin},{ }^{2}$ Shuang Xu, ${ }^{4}$ Fei Teng, ${ }^{5}$ Nengming Lin (10 ${ }^{6}$

To cite: Shi C, Ye J, Xu R, et al. Effect of the vancomycin minimum inhibitory concentration on clinical outcomes in patients with methicillin-susceptible Staphylococcus aureus bacteraemia: a systematic review and metaanalysis. BMJ Open 2021;11:e040675. doi:10.1136/ bmjopen-2020-040675

- Prepublication history and additional material is published online only. To view please visit the journal online (http://dx.doi. org/10.1136/bmjopen-2020040675).

$\mathrm{CS}, \mathrm{JY}$ and RX contributed equally.

Received 20 May 2020 Revised 11 December 2020 Accepted 21 December 2020

Check for updates

(C) Author(s) (or their employer(s)) 2021. Re-use permitted under CC BY-NC. No commercial re-use. See rights and permissions. Published by BMJ.

For numbered affiliations see end of article.

Correspondence to Dr Nengming Lin; Inm1013@zju.edu.cn

\section{ABSTRACT}

Objective The use of the vancomycin minimum inhibitory concentration (MIC) as a prognostic predictor in patients with methicillin-susceptible Staphylococcus aureus (MSSA) has been debated in the last decade. We performed a systematic review and meta-analysis to investigate whether an elevated vancomycin MIC is associated with a worse prognosis for patients with MSSA bacteraemia.

Design Systematic review and meta-analysis.

Data sources PubMed, Embase and the Cochrane Library were searched from inception to December 2019.

Eligibility criteria Randomised controlled trials or observational studies were considered eligible if they provided clinical outcomes of patients with MSSA bacteraemia, stratified by vancomycin MIC.

Data synthesis Primary outcome was mortality. Secondary outcomes included septic thrombophlebitis, persistent bacteraemia and complicated bacteraemia. Pooled ORs and 95\% Cls were calculated. Subgroup analyses included the susceptibility testing method. Results Fifteen observational studies were included. Bacteraemia due to MSSA isolates with high vancomycin MICs was associated with higher mortality than isolates with low MICs (OR 1.44; 95\% Cl 1.12 to $1.84 ; I^{2}=40.3 \%$ ). Additionally, significantly greater septic thrombophlebitis (OR 3.16; $95 \% \mathrm{Cl} 1.11$ to $9.00 ; \mathrm{I}^{2}=58.6 \%$ ) and a trend towards more persistent bacteraemia (OR 1.79; $95 \% \mathrm{Cl}$ 0.97 to $3.31 ;\left.\right|^{2}=0 \%$ ) were observed in patients with high vancomycin MICs than in patients with low MICs. Differences in complicated bacteraemia were not significant. Similar findings were obtained in subgroup analyses using Etest. However, significant differences in outcomes were not observed between the high and low vancomycin MICs detected using broth microdilution. Conclusion The available data suggest an association between elevated vancomycin MICs detected using Etest and adverse clinical outcomes for patients with MSSA bacteraemia. Future studies should validate these findings and explore the potential mechanisms. PROSPERO registration number CRD42018090547.
Strengths and limitations of this study

- This meta-analysis is the first study to comprehensively compare the clinical outcomes of patients with methicillin-susceptible Staphylococcus aureus bacteraemia with high and low vancomycin minimum inhibitory concentrations

- Two authors independently performed the study selection, data extraction and quality assessment.

- Numerous sensitivity analyses were performed to examine the robustness of the findings.

- Subgroup analyses based on the susceptibility testing method were also performed.

- Due to insufficient details of empirical and definitive treatments, a subgroup analysis based on the antibiotic treatment could not be performed.

\section{INTRODUCTION}

Staphylococcus aureus is one of the most common causes of healthcare-associated infections. Although more publications have focused on methicillin-resistant $S$. aureus (MRSA), bacteraemia caused by methicillinsusceptible $S$. aureus (MSSA) remains a significant global healthcare burden due to its high morbidity and mortality rates. ${ }^{1}$

The recognition of potential risk factors is considered vital to improve the management of patients with MSSA bacteraemia. According to previous studies, $\beta$-lactams are superior to vancomycin in terms of survival for the treatment of MSSA bacteraemia. $^{3-5}$ Therefore, the current guidelines recommend switching therapy to a $\beta$-lactam antibiotic, such as cefazolin or antistaphylococcal penicillins (ASPs), after the identification of an MSSA infection. ${ }^{6}$ Furthermore, our previous meta-analysis ${ }^{7}$ systematically 
evaluated studies focusing on the efficacy and safety of cefazolin versus ASPs in treating MSSA bacteraemia and found that cefazolin was significantly correlated with reductions in the death rate, clinical failure, hepatotoxicity and nephrotoxicity. The results favour cefazolin for the management of MSSA bacteraemia.

The choice of the antibiotic is a well-recognised risk factor for MSSA bacteraemia, but less is known about the effects of microbiological factors. A decade ago, two studies reported an association between a high vancomycin minimum inhibitory concentration (MIC) and a worse prognosis in terms of mortality and complicated bacteraemia among patients with MSSA bacteraemia, regardless of the antibiotic therapy used. ${ }^{89}$ In 2014, Kalil et $a l^{10}$ conducted a meta-analysis to identify a potential relationship between vancomycin MICs and mortality for patients with $S$. aureus bloodstream infections. Unfortunately, a subgroup analysis of MSSA bacteraemia was unable to be performed because insufficient publications were available at that time. Since then, a growing number of studies have focused on this interesting issue but have reported inconsistent results. Therefore, we conducted a systematic review and meta-analysis to assess the effect of the vancomycin MIC on clinical outcomes in patients with MSSA bacteraemia.

\section{METHODS}

\section{Literature search}

PubMed, Embase and the Cochrane Library were searched from inception to December 2019 by two investigators. The search terms and strategies for this metaanalysis are summarised in online supplemental table S1). References from identified publications and relevant reviews were also checked to identify additional articles. No language restriction was imposed.

\section{Inclusion criteria and study selection}

Two authors independently screened the literature, and discrepancies were resolved through discussion. Randomised controlled trials or observational studies were considered eligible if they provided clinical outcomes of patients with MSSA bacteraemia, stratified by vancomycin MIC. Broth microdilution (BMD), automated BMD (eg, Microscan), and Etest were considered reasonable susceptibility testing methods. The following types of studies were excluded: (1) the study population did not consist of patients with MSSA bacteraemia, (2) solely paediatric studies, (3) no outcome data were available, (4) duplicate publications and (5) conference abstracts.

\section{Data extraction and quality assessment}

Two reviewers independently extracted the data, and discrepancies were resolved by consensus. The following information was collected: study design, countries where the study was conducted, study period, sample size, patient characteristics (age, sex, comorbidity, severity of illness, and proportions of endocarditis), vancomycin MIC testing method, cut-off value, antimicrobial therapy used and the clinical outcomes. The Risk of Bias in Nonrandomized Studies for Interventions (ROBINS-I) tool was used to assess the quality of observational studies. ${ }^{11}$

\section{Definitions and outcomes}

The primary outcome was mortality. When data from more than one time point were available, the mortality at the latest point reporting the largest number of deaths was used in the main analysis (eg, 30-day mortality had precedence over 14-day mortality). The secondary outcomes included septic thrombophlebitis, persistent bacteraemia, and complicated bacteraemia, as defined by the individual. If vancomycin MIC was detected using multiple methods, the data from the reference BMD method were adopted in the main analysis. We did not define specific cut-off points for the vancomycin MIC because different criteria were used in various studies.

\section{Statistical analysis}

The ORs and 95\% CIs for outcomes were calculated. If adjusted ORs were provided in the included studies, then they were used for data combination. Otherwise, unadjusted ORs were used for data combination. Heterogeneity was assessed using the $\mathrm{I}^{2}$ statistic, and a value of $>50 \%$ was considered significant heterogeneity. ${ }^{12}$ The fixed effects model was used when significant heterogeneity was not present; otherwise, the random effects model was used. For the outcomes using fixed effects model, we also calculated the random effects model for additional analyses to provide conservative pooling estimates. Subgroup analyses were performed based on the susceptibility testing method (Etest and BMD). Sensitivity analyses were conducted for the primary outcome by removing each study individually. Additional sensitivity analyses were performed by restricting studies that were classified according to a specific criterion, such as prospective, large-sample $(\mathrm{n} \geq 90)$, high-quality (low to moderate risk of bias in 'overall assessment' of ROBIN-I tool), unadjusted mortality rate, adjusted mortality rate and 30-day mortality rate (the most frequent mortality rate reported). Publication bias was estimated by constructing a funnel plot and Egger's test. All the statistical analyses in the present meta-analysis were performed using STATA software V.15.0 (StataCorp, serial NO. 301506351850). The significance threshold was set to 0.05 .

\section{RESULTS}

\section{Search results}

Fifteen studies ${ }^{8913-25}$ involving 2487 participants met the inclusion criteria. The details regarding identification of relevant studies are shown in figure 1 . Two studies ${ }^{176}$ contained duplicate patients and the study with smaller sample size ${ }^{26}$ was excluded.

All studies were published within the last decade, and the number of patients per study ranged from 53 to 334 . 


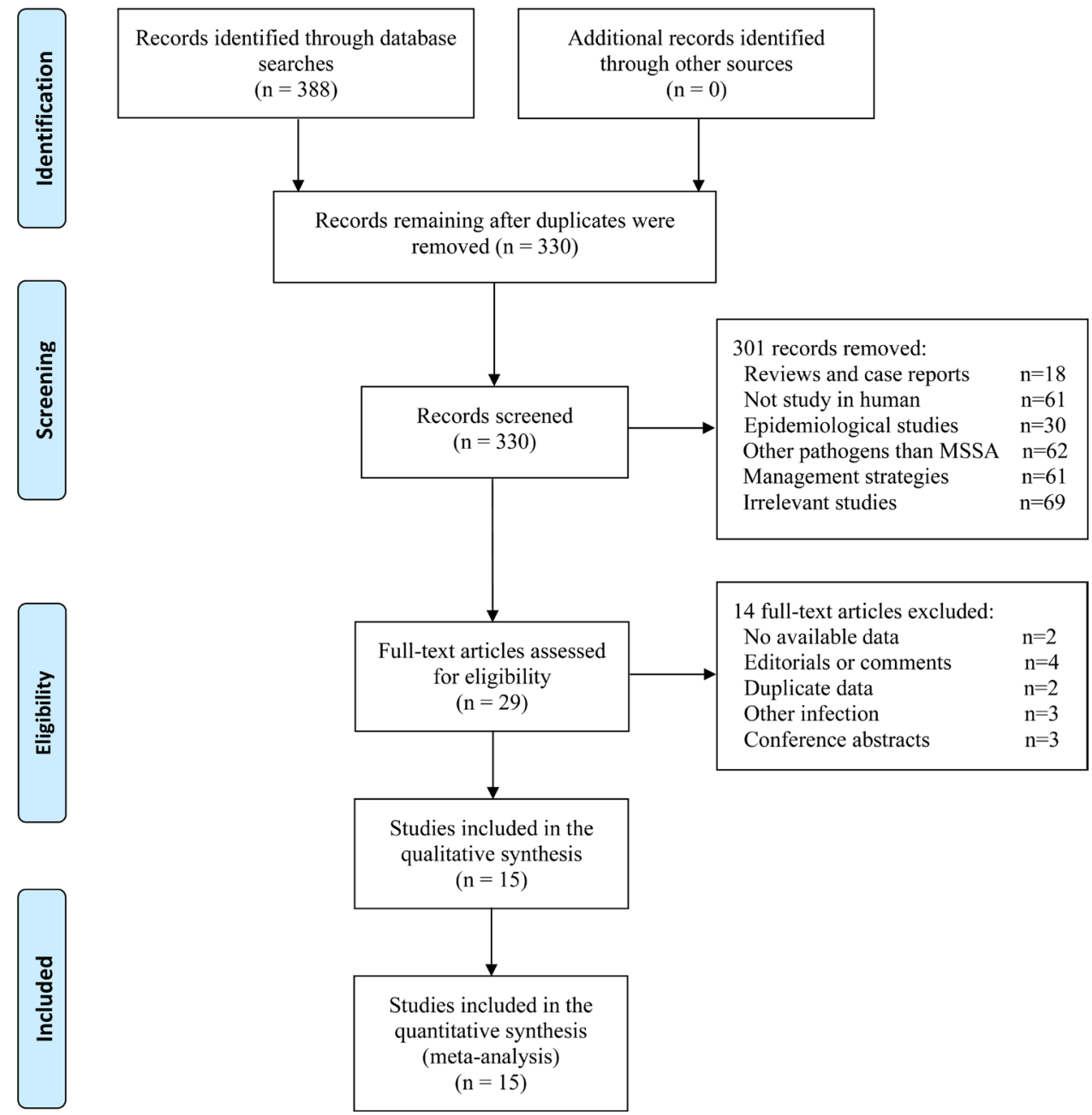

Figure 1 Flow diagram of the literature search and selection process. MSSA, methicillin-susceptible Staphylococcus aureus.

Eight studies were prospective observational studies, and the others were retrospective studies. The proportion of females ranged from $28 \%$ to $54.7 \%$, and the mean or median age ranged from 60 to 68 years. Most studies were conducted in Europe $(n=10)$, followed by the USA $(\mathrm{n}=3)$ and South Korea $(\mathrm{n}=1)$; the remaining study was conducted in multiple countries (Australia and New Zealand). Most of the studies used the Etest method to determine the vancomycin MIC and chose an MIC value $\geq 1.5 \mu \mathrm{g} / \mathrm{mL}$ as the cut-off for a high vancomycin MIC. Additional details of the characteristics are presented in table 1. Raw data for outcomes extracted from the included studies are presented in online supplemental table S2-S5). Risk-of-bias assessment showed that six studies ${ }^{13} 1518$ 22-24 had an overall critical risk of bias, and nine studies ${ }^{8914161719-2125}$ had an overall low to moderate risk of bias (online supplemental table S6).

\section{Primary outcome: mortality}

Fourteen studies reported mortality rates. ${ }^{8} 9$ 13-17 19-25 The mortality rates of MSSA bacteraemia varied among studies from $12 \%$ to $43 \%$ (table 1). Overall, the main analysis using a fixed effects model showed the mortality rate for patients infected with MSSA isolates with a high vancomycin MIC was significantly higher than isolates with a low MIC (OR 1.44; 95\% CI 1.12 to $1.84 ; \mathrm{I}^{2}=40.3 \%$; figure 2). The additional analysis using the random effects model showed similar results (OR 1.52; 95\% CI 1.08 to 2.12; $\mathrm{I}^{2}=40.3 \%$; table 2 ). Sensitivity analyses limited to 14 studies providing unadjusted mortality rates (OR 1.42 ; $95 \%$ CI 1.13 to $1.79 ; \mathrm{I}^{2}=36.0 \%$ ), 6 studies providing adjusted mortality rates (OR 1.74; 95\% CI 1.16 to 2.60; $\left.\mathrm{I}^{2}=47.9 \%\right)$ and 12 studies reporting 30 -day mortality rates (OR 1.44; $95 \%$ CI 1.09 to $1.92 ; \mathrm{I}^{2}=28.2 \%$; table 3 ) did not substantially alter the results of the main analysis. Similar results were obtained using the leave-one-out method (online supplemental table S7) and other prespecified sensitivity analyses (table 3 ).

\section{Secondary outcomes}

Secondary outcome data were available in nine studies, ${ }^{8} 13$ 15-18 212224 and the definitions are shown in online supplemental table S8. Six studies reported septic thrombophlebitis. ${ }^{815-172122}$ An increased risk of septic thrombophlebitis was observed in patients with a high vancomycin MIC compared with patients with a low MIC in the pooled analysis using the random effects model (OR 3.16; 95\% CI 1.11 to $9.00 ; \mathrm{I}^{2}=58.6 \%$; figure 3 ). Four 


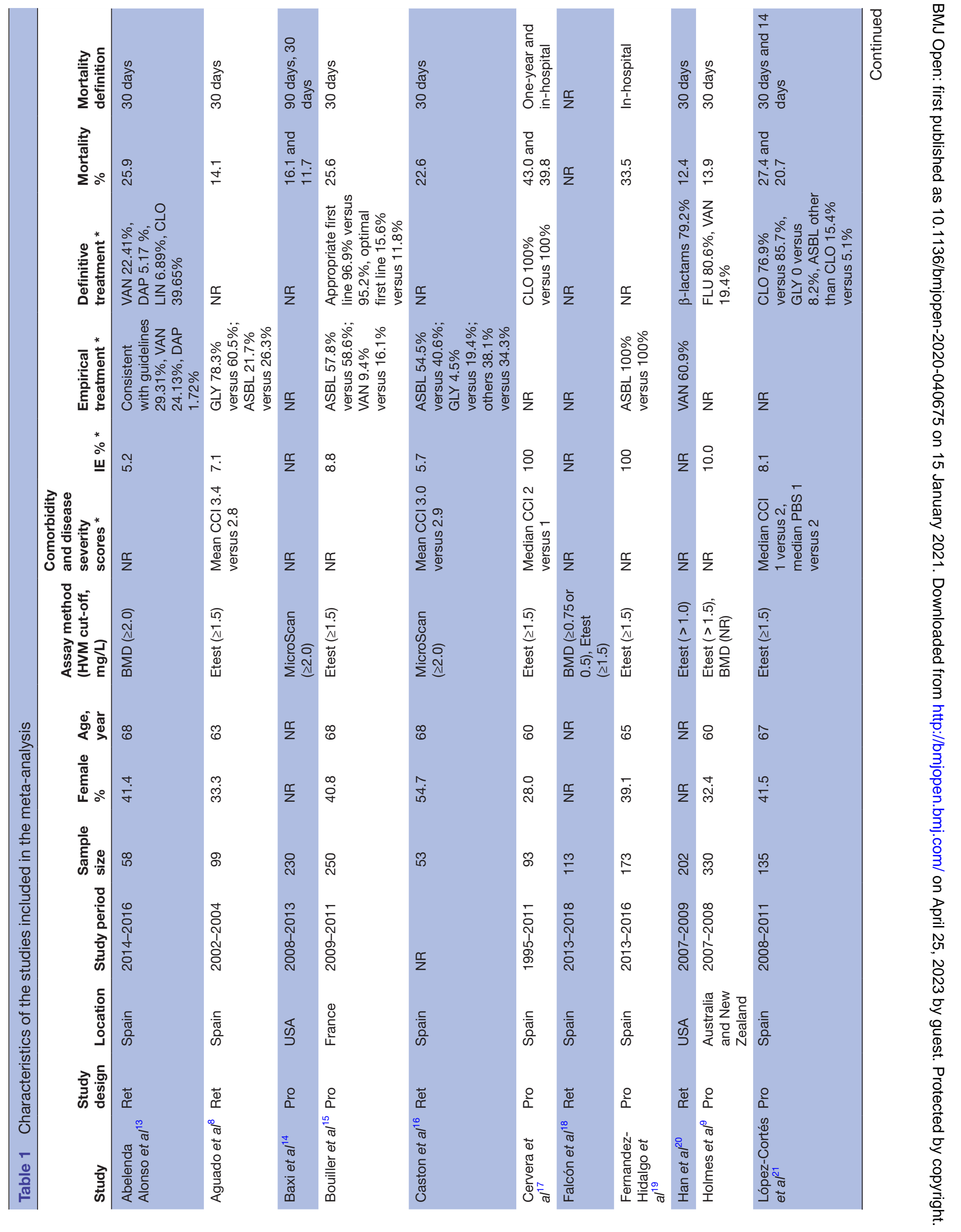




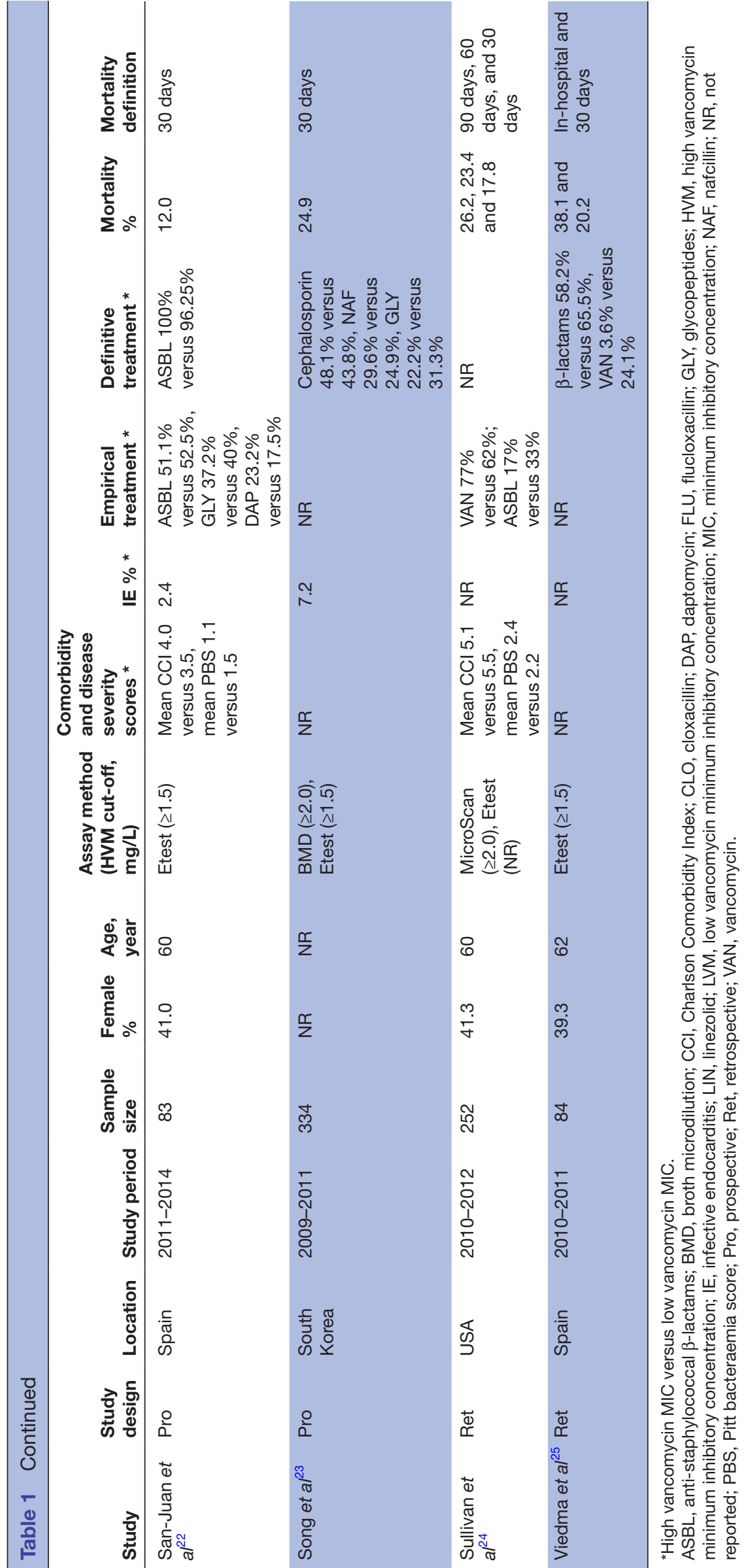




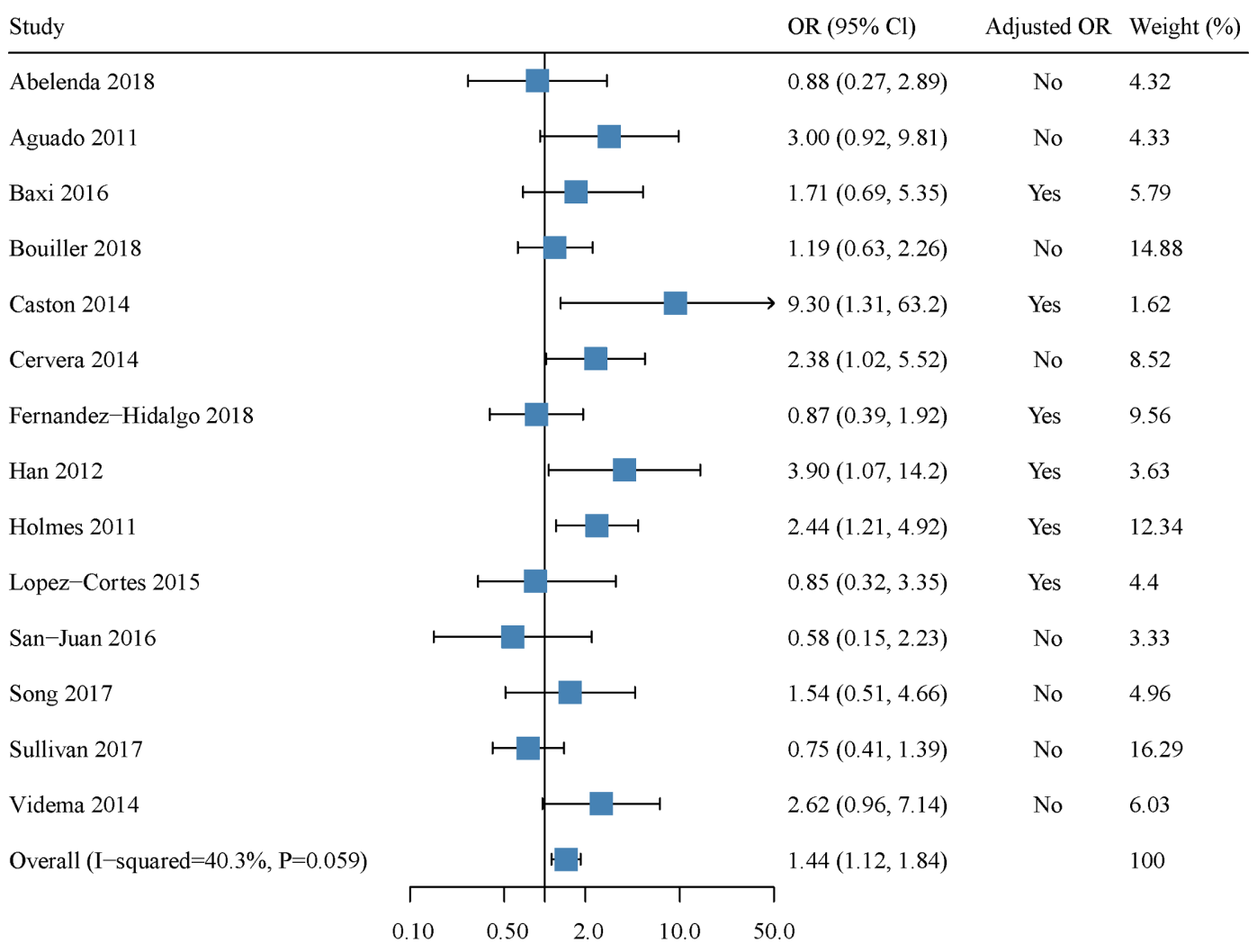

Figure 2 Forest plot of ORs for mortality.

studies reported persistent bacteraemia. ${ }^{13} 172122$ The meta-analysis using the fixed effects model revealed a non-significant higher risk of persistent bacteraemia in patients with a high vancomycin MIC (OR 1.79; 95\% CI 0.97 to $3.31 ; \mathrm{I}^{2}=0 \%$; figure 4 ). The additional analysis using the random effects model supported the main conclusions (table 2). Seven studies reported complicated

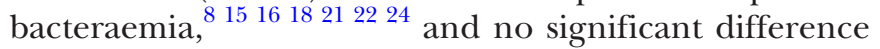

was observed in the pooled analysis using the random effects model (OR 1.59; 95\% CI 0.58 to $4.37 ; \mathrm{I}^{2}=87.0 \%$; figure 5).

\section{Subgroup analysis and publication bias}

In the subset of studies using Etest, patients with a high vancomycin MIC still exhibited a significantly higher mortality rate (OR 1.62; 95\% CI 1.21 to $2.17 ; \mathrm{I}^{2}=27.6 \%$ )

\section{Table 2 Subgroup analyses of outcomes}

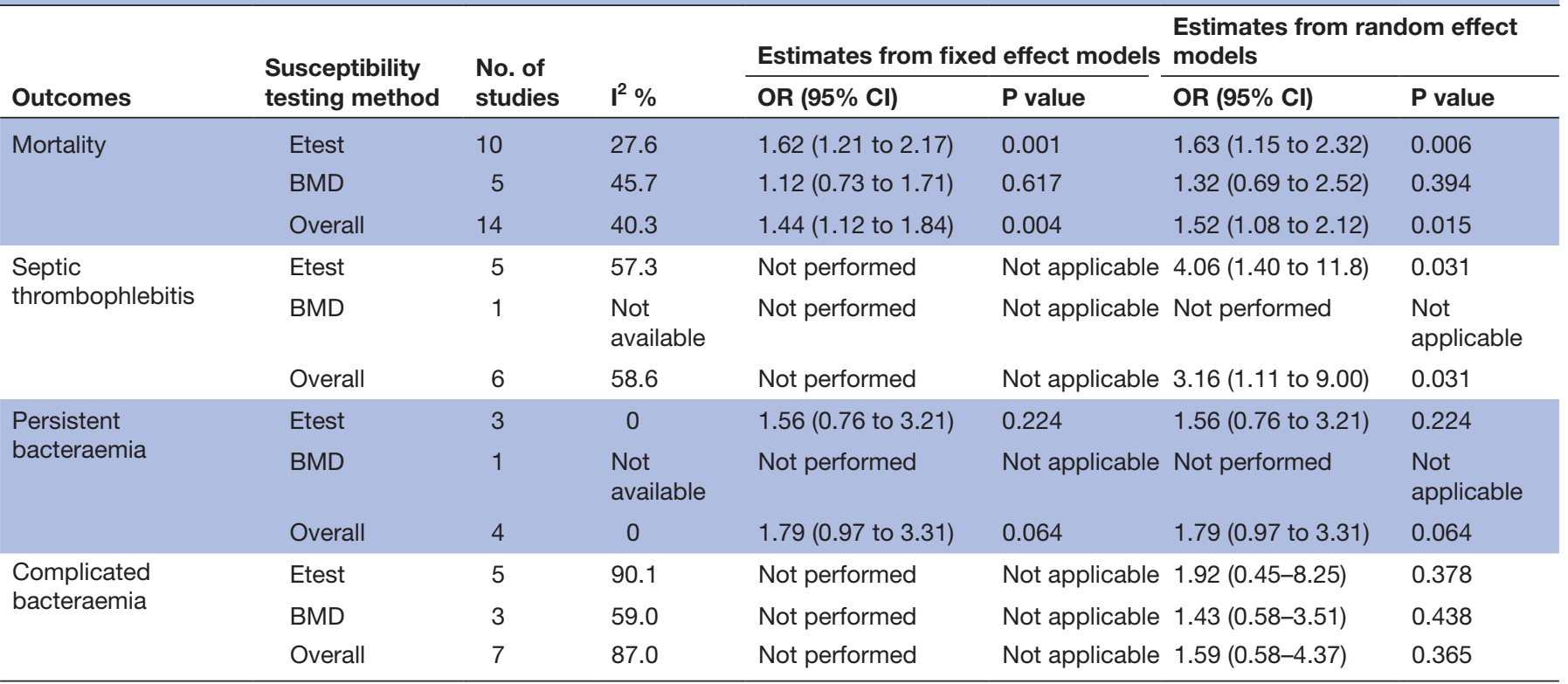

BMD, broth microdilution. 
Table 3 Sensitivity analyses of mortality

\begin{tabular}{|c|c|c|c|c|}
\hline Term included & No. of studies included & OR $(95 \% \mathrm{Cl})$ & $P$ value & $12 \%$ \\
\hline Prospective & 8 & 1.42 (1.04 to 1.93$)$ & 0.026 & 13.0 \\
\hline Large sample $(n \geq 90)$ & 10 & $1.48(1.04$ to 2.11$)$ & 0.012 & 37.6 \\
\hline Adjusted mortality & 6 & 1.74 (1.16 to 2.60$)$ & 0.007 & 47.9 \\
\hline Unadjusted mortality & 14 & 1.42 (1.13 to 1.79$)$ & 0.003 & 36.0 \\
\hline
\end{tabular}

and septic thrombophlebitis (OR 4.06; 95\% CI 1.40 to $11.80 ; \mathrm{I}^{2}=57.3 \%$ ) than patients with a low MIC. However, no significant differences in outcomes were observed between the high and low vancomycin MIC values detected using BMD (table 2). The results of the additional analyses using the random effects model supported the main conclusions (table 2). A visual inspection of the funnel plot showed symmetry (online supplemental figure S1), and the Egger test did not indicate significant publication bias $(\mathrm{p}=0.546)$.

\section{DISCUSSION}

To our knowledge, this systematic review and meta-analysis are the first to comprehensively compare the clinical outcomes of patients with MSSA bacteraemia presenting high and low vancomycin MICs. The main findings of the present study indicate that an elevated vancomycin MIC correlates with a higher mortality rate. The findings were relatively robust and were not significantly changed during the sensitivity analyses. In addition, patients with a high vancomycin MIC had a significantly higher risk of septic thrombophlebitis and a trend towards more persistent bacteraemia. No significant difference was observed in complicated bacteraemia. Based on these findings, the vancomycin MIC is potentially useful to predict adverse outcomes in patients with MSSA bacteraemia.
Several meta-analyses exploring the effect of the vancomycin MIC on the mortality rate in patients with a MRSA infection have produced positive correlations; the failure to achieve the pharmacokinetic/pharmacodynamic targets of vancomycin may be the main explanation. ${ }^{27-29}$ However, vancomycin is not routinely used in clinical practice after the identification of an MSSA infection. In fact, the proportion of patients who received vancomycin for definitive therapy is less than one quarter in the included studies (table 1). By analysing the subset of patients with MSSA bacteraemia treated with flucloxacillin, Holmes et al observed a significant correlation between the 30-day mortality rate and a high vancomycin MIC. ${ }^{9}$ Similar results were reported in the included study by Cervera $e t$ al, which included patients who exclusively received cloxacillin. ${ }^{17}$ We hypothesise that a decreased vancomycin susceptibility might be a surrogate marker of a weak response to both vancomycin and other antibiotics (eg, $\beta$-lactams) in the treatment of MSSA infections. Interestingly, MSSA isolates with decreased susceptibility to vancomycin also exhibit less susceptibility to the ASP and daptomycin. ${ }^{30}$ This phenomenon might have contributed to special structural modifications in the cell walls of isolates with an elevated vancomycin MIC, particularly an increased cell wall thickness. ${ }^{31}$ Moreover, reductions in autolysin activity and in the cell wall content of

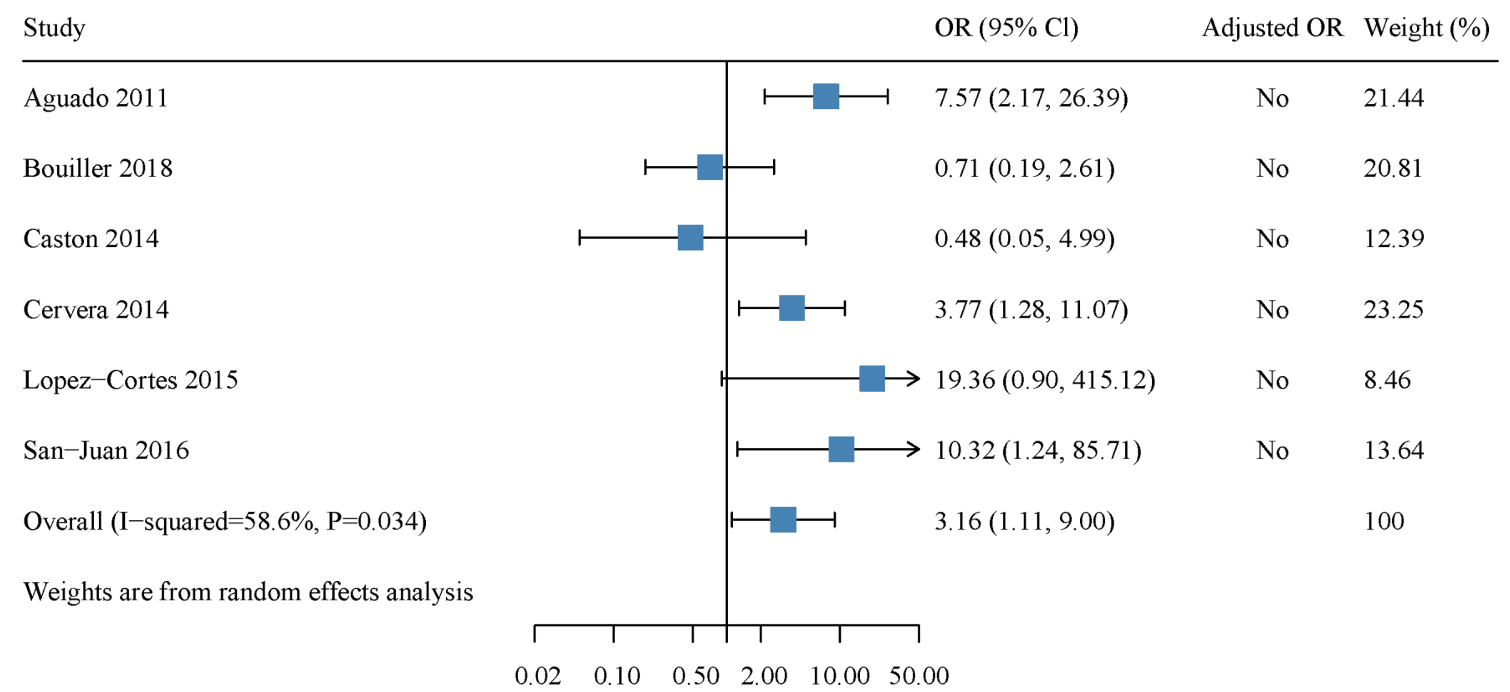

Figure 3 Forest plot of ORs for septic thrombophlebitis. 


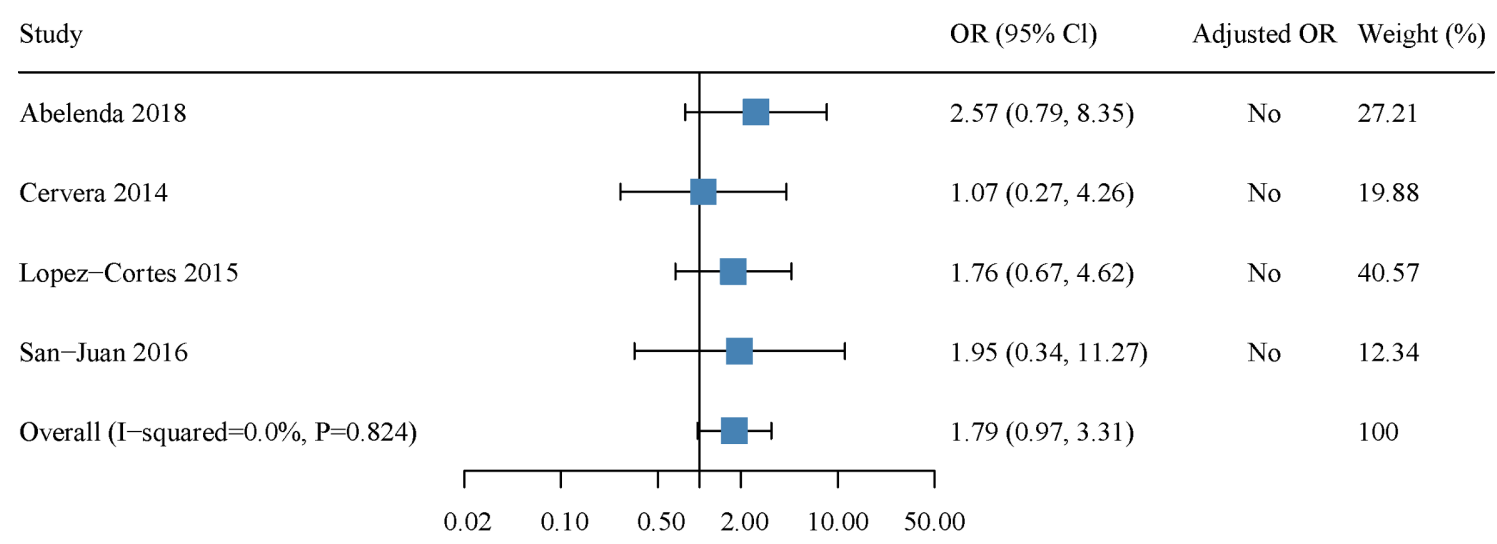

Figure 4 Forest plot of ORs for persistent bacteraemia.

penicillin binding protein 4 may be involved in these changes. $^{32}$

The elevated vancomycin MIC might also be a marker for unfavourable bacterial or genetic factors that lead to adverse outcomes in patients with MSSA bacteraemia. The accessory gene regulator $(a g r)$ is a quorum-sensing regulator that is responsible for the expression of various virulence factors. ${ }^{33} \mathrm{Agr}$ dysfunction and agr type II have been correlated with an elevated vancomycin MIC, increased mortality rate, more persistent bacteraemia and increased biofilm formation. ${ }^{25}$ 34-37 Moreover, we noticed that agr type III was less prevalent in MSSA isolates with a high vancomycin MIC than in isolates with a low MIC $(17.2 \%$ vs $34.0 \%$ ) in the study by Lopez-Cortes $e t a l,{ }^{21}$ although this difference did not reach statistical significance. In fact, the negative correlation between agr type III and mortality was observed in MSSA infective endocarditis. ${ }^{19}$

Multiple clonal complexes (CCs), including CC5, CC8, CC88 and CC188, are associated with reduced vancomycin susceptibility in S. aureus bacteraemia isolates. ${ }^{25} 34$ A recent study ${ }^{38}$ analysed the genotypic profiles among 93 MSSA isolates reported in the study by Cervera $e^{~} \mathrm{al}^{17}$ and found that CC5 was significantly correlated with a higher mortality rate in the univariate analysis. Another included study ${ }^{19}$ also revealed a correlation between MSSA isolates harbouring CC8 and increased in-hospital mortality in the univariate analysis.

Furthermore, specific resistance and virulence genes (including blaZ, sea, clfA, and splA) and the arginine catabolic mobile element locus were linked with an elevated vancomycin MIC in S. aureus bacteraemia. ${ }^{34}$ MSSA isolates with genotypes $s p a-002$ and $s p a-\mathrm{CC} 008$ correlate with an elevated vancomycin $\mathrm{MIC},{ }^{24}$ and isolates with extracellular fibrinogen binding protein and serine endopeptidase are associated with a higher incidence of embolism. ${ }^{26}$

Overall, the mechanism underlying the association between the increased vancomycin MIC and adverse outcomes in patients with MSSA bacteraemia appear to be complex. We postulate that this correlation is not attributed to a single cause, but results from the interactive effects of multiple factors. The findings of the present meta-analysis highlight the need for further studies to fill the knowledge gap about this interesting issue.

Study
Aguado 2011
Bouiller 2018
Caston 2014
Falcon 2019
Lopez-Cortes 2015
San-Juan 2016
Sullivan 2017
Overall (I-squared=87.0\%, $\mathrm{P}=0.000)$
Weights are from random effects analysis

Figure 5 Forest plot of ORs for complicated bacteraemia. 
Notably, the most frequently used test to detect the vancomycin MIC in the included studies was the Etest. We identified a few studies performed susceptibility testing using Etest in parallel with manual or automated BMD methods. According to Han et al, the vancomycin MIC detected using Etest poorly correlated with the values obtained using the BMD method. ${ }^{20}$ Song et al reported the consistent underestimation of the MIC values from Etest among isolates with a high vancomycin MIC $(2 \mathrm{mg} / \mathrm{L})$ and overestimated among isolates with a low MIC $(0.5 \mathrm{mg} / \mathrm{L})$ using BMD.$^{23}$ As shown in the study by Sullivan et al, the vancomycin MIC values measured using automated BMD (Microscan) are consistently higher than the values measured using Etest in the majority of MSSA isolates. ${ }^{24}$ Because the application of the various susceptibility testing methods may introduce bias, we conducted a subgroup analysis according to the MIC methodology. In the subgroup analysis of studies using Etest, patients with a high vancomycin MIC still had a significantly higher mortality rate than patients with a low MIC. However, no significant difference was observed in the subgroup analysis of studies using BMD. Notably, we did not include the study by Holmes et al in the subgroup analysis of studies using BMD due to the unavailability of data, although the authors stated that a higher mortality rate was correlated with an increased vancomycin MIC detected using BMD. Therefore, the results of the subgroup analysis of studies using BMD may be underestimated. Nevertheless, the present meta-analysis indicated that Etest might be better for predicting clinical outcomes, although the reproducibility and interobserver variation may be a concern, as noted by Falcón et $a l^{39}$ Because the BMD is still the reference method for vancomycin susceptibility testing, more studies using the BMD are needed to validate the association between vancomycin MIC and clinical outcomes.

The present meta-analysis has several limitations that should be considered when interpreting the results. First, all studies included in the meta-analysis were observational studies containing many confounding factors. However, only six out of the included studies were designed to adjust for confounding factors and reported adjusted mortality rates. For studies without adjustment for potential confounders, the baseline characteristics of the patients (eg, underlying conditions and the calculation of severity scores) between the high and low vancomycin MIC groups may influence the outcomes. For example, patients with high vancomycin MICs had numerically higher mean or median CCI scores in several studies. ${ }^{172}$ Bouiller et al observed more dialysis and a higher proportion of ultimately fatal disease in the high vancomycin MIC group. ${ }^{15}$ More underlying diseases and higher CCI scores might be associated with adverse outcomes. Therefore, the adverse effect of the high vancomycin MIC on clinical outcomes might be overestimated. Second, almost half of the studies were retrospective in design, which may introduce inherent selection bias into this report. To address the potential confounding bias and selection bias, numerous sensitivity analyses were performed. Sensitivity analyses limited to prospective studies, high-quality studies based on the
ROBIN-I tool, and studies providing adjusted mortality data did not substantially alter the results of the main analysis, further supporting the conclusions in our study. Third, as we mentioned in the Introduction section, the choice of antibiotic is closely related to the survival of patients with MSSA bacteraemia. However, the dose and duration of antibiotic therapy and the types of $\beta$-lactams chosen were not reported in most included studies. Due to insufficient details regarding both empirical and definitive treatments, a subgroup analysis based on the antibiotic treatment was unable to be conducted. Moreover, our previous findings regarding the differences in efficacy and safety between cefazolin and ASPs have not been sufficiently considered in the included studies. ${ }^{7}$ Fourth, most MSSA isolates in the included studies had a vancomycin MIC $\leq 2 \mathrm{mg} / \mathrm{L}$ determined using BMD or $\leq 1.5 \mathrm{mg} / \mathrm{L}$ determined using Etest, and the outcomes of MSSA bacteraemia caused by more resistant isolates are unclear.

In conclusion, the available data suggest a correlation between a high vancomycin MIC detected using Etest and adverse clinical outcomes for patients with MSSA bacteraemia. More well-designed studies are warranted to validate the present findings and to explore the potential mechanisms.

\section{Author affiliations}

${ }^{1}$ Department of Clinical Pharmacy, Key Laboratory of Clinical Cancer Pharmacology and Toxicology Research of Zhejiang Province, Affiliated Hangzhou First People's Hospital, Zhejiang University School of Medicine, Hangzhou, Zhejiang, China ${ }^{2}$ Department of Respiratory Medicine, Affiliated Hangzhou First People's Hospital, Zhejiang University School of Medicine, Hangzhou, Zhejiang, China

${ }^{3}$ Department of Clinical Pharmacy, Shaoxing Women and Children's Hospital, Shaoxing, Zhejiang, China

${ }^{4}$ Translational Medicine Research Center, Hangzhou First People's Hospital, Nanjing Medical University, Hangzhou, Zhejiang, China

${ }^{5}$ College of Pharmaceutical Sciences, Zhejiang Chinese Medical University, Hangzhou, Zhejiang, China

${ }^{6}$ Department of Clinical Pharmacology, Translational Medicine Research Center, Key Laboratory of Clinical Cancer Pharmacology and Toxicology Research of Zhejiang Province, Affiliated Hangzhou First People's Hospital, Zhejiang University School of Medicine, Hangzhou, Zhejiang, China

Contributors NL contributed to conception and design. All authors contributed to acquisition, analysis and interpretation of data. CS was involved in drafting or critical revision of the manuscript. All authors approved the final version.

Funding This study was supported by Hangzhou Health Science and Technology Planning Project (grant number: 0020190083).

Competing interests None declared.

Patient consent for publication Not required.

Provenance and peer review Not commissioned; externally peer reviewed.

Data availability statement All data relevant to the study are included in the article or uploaded as supplementary information.

Supplemental material This content has been supplied by the author(s). It has not been vetted by BMJ Publishing Group Limited (BMJ) and may not have been peer-reviewed. Any opinions or recommendations discussed are solely those of the author(s) and are not endorsed by BMJ. BMJ disclaims all liability and responsibility arising from any reliance placed on the content. Where the content includes any translated material, BMJ does not warrant the accuracy and reliability of the translations (including but not limited to local regulations, clinical guidelines, terminology, drug names and drug dosages), and is not responsible for any error and/or omissions arising from translation and adaptation or otherwise.

Open access This is an open access article distributed in accordance with the Creative Commons Attribution Non Commercial (CC BY-NC 4.0) license, which 
permits others to distribute, remix, adapt, build upon this work non-commercially, and license their derivative works on different terms, provided the original work is properly cited, appropriate credit is given, any changes made indicated, and the use is non-commercial. See: http://creativecommons.org/licenses/by-nc/4.0/.

\section{ORCID iD}

Nengming Lin http://orcid.org/0000-0001-5202-802X

\section{REFERENCES}

1 Laupland KB, Lyytikäinen O, Søgaard M, et al. The changing epidemiology of Staphylococcus aureus bloodstream infection: a multinational population-based surveillance study. Clin Microbiol Infect 2013:19:465-71.

2 Cosgrove SE, Sakoulas G, Perencevich EN, et al. Comparison of mortality associated with methicillin-resistant and methicillinsusceptible Staphylococcus aureus bacteremia: a meta-analysis. Clin Infect Dis 2003;36:53-9.

3 McDanel JS, Perencevich EN, Diekema DJ, et al. Comparative effectiveness of beta-lactams versus vancomycin for treatment of methicillin-susceptible Staphylococcus aureus bloodstream infections among 122 hospitals. Clin Infect Dis 2015;61:361-7.

4 Schweizer ML, Furuno JP, Harris AD, et al. Comparative effectiveness of nafcillin or cefazolin versus vancomycin in methicillin-susceptible Staphylococcus aureus bacteremia. BMC Infect Dis 2011;11:279.

$5 \mathrm{Kim} \mathrm{S-H,} \mathrm{Kim} \mathrm{K-H,} \mathrm{Kim} \mathrm{H-B,} \mathrm{et} \mathrm{al.} \mathrm{Outcome} \mathrm{of} \mathrm{vancomycin}$ treatment in patients with methicillin-susceptible Staphylococcus aureus bacteremia. Antimicrob Agents Chemother 2008;52:192-7.

6 Liu C, Bayer A, Cosgrove SE, et al. Clinical practice guidelines by the infectious diseases Society of America for the treatment of methicillin-resistant Staphylococcus aureus infections in adults and children. Clin Infect Dis 2011;52:e18-55.

7 Shi C, Xiao Y, Zhang Q, et al. Efficacy and safety of cefazolin versus antistaphylococcal penicillins for the treatment of methicillinsusceptible Staphylococcus aureus bacteremia: a systematic review and meta-analysis. BMC Infect Dis 2018;18:508.

8 Aguado JM, San-Juan R, Lalueza A, et al. High vancomycin MIC and complicated methicillin-susceptible Staphylococcus aureus bacteremia. Emerg Infect Dis 2011;17:1099-102.

9 Holmes NE, Turnidge JD, Munckhof WJ, et al. Antibiotic choice may not explain poorer outcomes in patients with Staphylococcus aureus bacteremia and high vancomycin minimum inhibitory concentrations. $J$ Infect Dis 2011;204:340-7.

10 Kalil AC, Van Schooneveld TC, Fey PD, et al. Association between vancomycin minimum inhibitory concentration and mortality among patients with Staphylococcus aureus bloodstream infections: a systematic review and meta-analysis. JAMA 2014;312:1552-64.

11 Sterne JA, Hernán MA, Reeves BC, et al. ROBINS-I: a tool for assessing risk of bias in non-randomised studies of interventions. BMJ 2016;355:i4919.

12 Higgins JPT, Thompson SG. Quantifying heterogeneity in a metaanalysis. Stat Med 2002;21:1539-58.

13 Abelenda Alonso G, Corbacho Loarte MD, Núñez Ramos R, et al. Staphylococcus aureus bacteremia in a secondary level Spanish Hospital: clinical implications of high vancomycin MIC. Rev Esp Quimioter 2018;31:353-62.

14 Baxi SM, Clemenzi-Allen A, Gahbauer A, et al. Vancomycin MIC does not predict 90 -day mortality, readmission, or recurrence in a prospective cohort of adults with Staphylococcus aureus bacteremia. Antimicrob Agents Chemother 2016;60:5276-84.

15 Bouiller K, Laborde C, Aho SL, et al. No effect of vancomycin $\mathrm{MIC} \geq 1.5 \mathrm{mg} / \mathrm{L}$ on treatment outcome in methicillin-susceptible Staphylococcus aureus bacteraemia. Int $J$ Antimicrob Agents 2018;51:721-6.

16 Castón JJ, González-Gasca F, Porras L, et al. High vancomycin minimum inhibitory concentration is associated with poor outcome in patients with methicillin-susceptible Staphylococcus aureus bacteremia regardless of treatment. Scand J Infect Dis 2014;46:783-6.

17 Cervera C, Castañeda X, de la Maria CG, et al. Effect of vancomycin minimal inhibitory concentration on the outcome of methicillinsusceptible Staphylococcus aureus endocarditis. Clin Infect Dis 2014;58:1668-75.

18 Falcón R, Mateo E, Oltra R, et al. Vancomycin MICs and risk of complicated bacteremia by glycopeptide-susceptible Staphylococcus aureus. Eur J Clin Microbiol Infect Dis 2019;38:903-12

19 Fernández-Hidalgo N, Ribera A, Larrosa MN, et al. Impact of Staphylococcus aureus phenotype and genotype on the clinical characteristics and outcome of infective endocarditis. A multicentre, longitudinal, prospective, observational study. Clin Microbiol Infect 2018;24:985-91.

20 Han JH, Mascitti KB, Edelstein $\mathrm{PH}$, et al. Effect of reduced vancomycin susceptibility on clinical and economic outcomes in Staphylococcus aureus bacteremia. Antimicrob Agents Chemother 2012;56:5164-70.

21 López-Cortés LE, Velasco C, Retamar P, et al. Is reduced vancomycin susceptibility a factor associated with poor prognosis in MSSA bacteraemia? J Antimicrob Chemother 2015;70:2652-60.

22 San-Juan R, Viedma E, Chaves F, et al. High MICs for vancomycin and daptomycin and complicated catheter-related bloodstream infections with methicillin-sensitive Staphylococcus aureus. Emerg Infect Dis 2016;22:1057-66.

23 Song K-H, Kim M, Kim CJ, et al. Impact of vancomycin MIC on treatment outcomes in invasive Staphylococcus aureus infections. Antimicrob Agents Chemother 2017;61:e01845-16.

24 Sullivan SB, Austin ED, Stump S, et al. Reduced vancomycin susceptibility of methicillin-susceptible Staphylococcus aureus has no significant impact on mortality but results in an increase in complicated infection. Antimicrob Agents Chemother 2017;61:e00316-7.

25 Viedma E, Sanz F, Orellana MA, et al. Relationship between agr dysfunction and reduced vancomycin susceptibility in methicillinsusceptible Staphylococcus aureus causing bacteraemia. $J$ Antimicrob Chemother 2014;69:51-8.

26 Pericàs JM, Messina JA, Garcia-de-la-Mària C, et al. Influence of vancomycin minimum inhibitory concentration on the outcome of methicillin-susceptible Staphylococcus aureus left-sided infective endocarditis treated with antistaphylococcal $\beta$-lactam antibiotics: a prospective cohort study by the International collaboration on endocarditis. Clin Microbiol Infect 2017;23:544-9.

27 Jacob JT, DiazGranados CA. High vancomycin minimum inhibitory concentration and clinical outcomes in adults with methicillinresistant Staphylococcus aureus infections: a meta-analysis. Int $J$ Infect Dis 2013;17:e93-100.

28 van Hal SJ, Lodise TP, Paterson DL. The clinical significance of vancomycin minimum inhibitory concentration in Staphylococcus aureus infections: a systematic review and meta-analysis. Clin Infect Dis 2012;54:755-71.

29 Mavros MN, Tansarli GS, Vardakas KZ, et al. Impact of vancomycin minimum inhibitory concentration on clinical outcomes of patients with vancomycin-susceptible Staphylococcus aureus infections: a meta-analysis and meta-regression. Int $\mathrm{J}$ Antimicrob Agents 2012;40:496-509.

30 Pillai SK, Wennersten C, Venkataraman L, et al. Development of reduced vancomycin susceptibility in methicillin-susceptible Staphylococcus aureus. Clin Infect Dis 2009;49:1169-74.

31 Falcón R, Martínez A, Albert E, et al. High vancomycin MiCs within the susceptible range in Staphylococcus aureus bacteraemia isolates are associated with increased cell wall thickness and reduced intracellular killing by human phagocytes. Int $J$ Antimicrob Agents 2016;47:343-50.

32 Mimica MJ. Reduced susceptibility to vancomycin in Staphylococcus aureus. Emerg Infect Dis 2011;17:2083-4.

33 Bronner S, Monteil H, Prévost G. Regulation of virulence determinants in Staphylococcus aureus: complexity and applications. FEMS Microbiol Rev 2004;28:183-200.

34 Holmes NE, Turnidge JD, Munckhof WJ, et al. Genetic and molecular predictors of high vancomycin MIC in Staphylococcus aureus bacteremia isolates. J Clin Microbiol 2014;52:3384-93.

35 Schweizer ML, Furuno JP, Sakoulas G, et al. Increased mortality with accessory gene regulator (agr) dysfunction in Staphylococcus aureus among bacteremic patients. Antimicrob Agents Chemother 2011;55:1082-7.

36 Park S-Y, Chong YP, Park HJ, et al. Agr dysfunction and persistent methicillin-resistant Staphylococcus aureus bacteremia in patients with removed eradicable foci. Infection 2013;41:111-9.

37 Beenken KE, Mrak LN, Griffin LM, et al. Epistatic relationships between sarA and agr in Staphylococcus aureus biofilm formation. PLoS One 2010;5:e10790.

38 Pericàs JM, Cervera C, Garcia-de-la-Mària C, et al. Relationship between vancomycin MIC and virulence gene expression in clonal complexes of methicillin-susceptible Staphylococcus aureus strains isolated from left-sided endocarditis. Antimicrob Agents Chemother 2020;64:e01579-19.

39 Falcón R, Madrid S, Tormo N, et al. Intra- and interinstitutional evaluation of an Etest for vancomycin minimum inhibitory concentration measurement in Staphylococcus aureus blood isolates. Clin Infect Dis 2015;61:1490-2. 